\title{
Hepatocellular carcinoma diagnosis using a novel electrochemiluminescence immunoassay targeting serum IgM-free AIM
}

\author{
Tomo Shimizu ${ }^{1}$. Takashi Sawada ${ }^{1}$ Tomohide Asai ${ }^{1}$. Yuka Kanetsuki ${ }^{1}$. Jiro Hirota ${ }^{1}$ - Michihisa Moriguchi ${ }^{2}$. \\ Tomoaki Nakajima $^{3} \cdot$ Toru Miyazaki $^{4,5,6,7} \cdot$ Takeshi Okanoue $^{8}$
}

Received: 21 July 2021 / Accepted: 29 November 2021 / Published online: 4 January 2022

(c) The Author(s) 2021, corrected publication 2022

\begin{abstract}
Background Recent increases in the number of patients with non-alcoholic steatohepatitis (NASH) warrant the identification of biomarkers for early detection of hepatocellular carcinoma (HCC) associated with NASH (NASH-HCC). IgM-free apoptosis inhibitor of macrophage (AIM), which generally associates with IgM in blood and exerts its biological function by dissociation from IgM, may serve as an effective biomarker for NASH-HCC. Here, we established a fully automatic and high-throughput electrochemiluminescence immunoassay (ECLIA) to measure IgM-free AIM and investigated its efficacy in diagnosing NASH-HCC and viral HCC.

Methods IgM-free AIM levels were measured in 212 serum samples from patients with, or without, HCC related to NASH, hepatitis B virus, and hepatitis C virus, using ECLIA. We also developed an ECLIA for measuring both IgM-free and IgMbound AIM and investigated the existing form of AIM in blood by size-exclusion chromatography.

Results IgM-free AIM levels were significantly higher in the HCC group than in the non-HCC group, regardless of the associated pathogenesis. Moreover, the area under the receiver operating curve for IgM-free AIM was greater than that for conventional HCC biomarkers, alpha-fetoprotein or des- $\gamma$-carboxy prothrombin, regardless of the cancer stage. ECLIA counts of IgM-free AIM derived from samples fractionated by size-exclusion chromatography were significantly higher in patients with NASH-HCC than in healthy volunteers and in patients with non-alcoholic fatty liver and NASH.

Conclusions Serum IgM-free AIM may represent a universal HCC diagnostic marker superior to alpha-fetoprotein or des$\gamma$-carboxy prothrombin. Our newly established ECLIA could contribute to further clinical studies on AIM and in vitro HCC diagnosis.
\end{abstract}

Keywords Non-alcoholic steatohepatitis · Hepatocellular carcinoma · IgM-free apoptosis inhibitor of macrophage · Electrochemiluminescence immunoassay $\cdot$ Diagnosis

Tomo Shimizu

tomo_shimizu@sekisui.com

1 Tsukuba Research Institute Research and Development Division, Sekisui Medical Co., Ltd., 3-3-1, Koyodai, Ryugasaki, Ibaraki 301-0852, Japan

2 Department of Molecular Gastroenterology and Hepatology, Graduate School of Medical Science, Kyoto Prefectural University of Medicine, Kyoto 602-8566, Japan

3 Department of Hepatology, Sapporo Kosei General Hospital, Hokkaido 060-0033, Japan

4 Laboratory of Molecular Biomedicine for Pathogenesis, Center for Disease Biology and Integrative Medicine, Faculty of Medicine, The University of Tokyo, Tokyo 113-0033, Japan
5 LEAP, Japan Agency for Medical Research and Development, Tokyo 113-0033, Japan

6 Laboratoire d'ImmunoRhumatologie Moléculaire, Plateforme GENOMAX, Institut National de la Santé et de la Recherche Médicale UMR_S 1109, Faculté de Médecine, Fédération Hospitalo-Universitaire OMICARE, Fédération de Médecine Translationnelle de Strasbourg, Laboratory of Excellence TRANSPLANTEX, Université de Strasbourg, Strasbourg, France

7 The Institute for AIM Medicine, Tokyo 101-0047, Japan

8 Department of Gastroenterology and Hepatology, Saiseikai Suita Hospital, Osaka 564-0013, Japan 


\section{Introduction}

Non-alcoholic fatty liver disease (NAFLD) is the most common chronic liver disease in many countries and is closely associated with metabolic syndrome [1-4]. NAFLD is classified as non-alcoholic fatty liver (NAFL) (benign form) and non-alcoholic steatohepatitis (NASH) (active form), which could progress to cirrhosis and hepatocellular carcinoma (HCC) [5]. Most HCCs are caused by viral hepatitis; however, therapeutic advances have contributed to viral clearance in many patients with hepatitis $\mathrm{C}$, while those with hepatitis $\mathrm{B}$ are often clinically controlled using nucleotide/nucleoside analogs $[6,7]$. Consequently, the annual incidence of hepatitis virus-induced HCC has gradually decreased. In contrast, the number of patients with obesity and diabetes has increased; as such, NASH-HCC incidence has increased against the backdrop of metabolic syndrome NASH associated with obesity, diabetes, dyslipidemia, and hypertension, necessitating the development of appropriate countermeasures that can be applied worldwide [8].

Alpha-fetoprotein (AFP) and des- $\gamma$-carboxy prothrombin (DCP) are widely recognized HCC biomarkers. For early HCC detection, surveillance comprising a combination of abdominal ultrasonography with multiple biomarkers is recommended in patients with chronic liver disease, particularly in those with cirrhosis derived from advanced fibrosis. However, recent studies have shown that $1 / 4$ to $1 / 3$ of patients with NASH develop HCC without cirrhosis $[9,10]$. Furthermore, we previously reported that serum AFP is not significantly increased in most patients with NASH-HCC [10]. In contrast, serum IgM-free apoptosis inhibitor of macrophage (AIM) is a useful biomarker of NASH-HCC, irrespective of AFP or DCP status [11].

AIM, a secretory glycoprotein produced by macrophages, suppresses macrophage apoptosis [12]. AIM comprises three scavenger receptor cysteine-rich domains with a molecular weight of approximately $40 \mathrm{kDa}$. In blood, AIM combines with high-molecular weight IgM pentamers at a 1:1 equimolar ratio through a disulfide bond and a charge-based interaction between AIM and IgM Fc, and exists in a stabilized, inactive state [13, 14]. However, it exhibits various biological activities as IgM-free AIM, following its release from IgM [15]. For instance, animal experiments have demonstrated its lipolytic activity in adipocytes and hepatocytes, anti-liver cancer activity based on activation of complement-dependent cytotoxicity, and cell residue removal activity in renal tubules [16-18]. Thus, AIM may be considered a therapeutic target for obesity, fatty liver, liver cancer, and acute kidney injury. In the liver, AIM is taken up by cells via CD36 where it demonstrates lipolytic activity in normal cells; however, in cancer cells, cellular uptake is suppressed, and AIM accumulates on the cell surface, activating complement proteins and causing subsequent cancer cell death [17].

To use AIM as a tumor marker, it is necessary to establish a method capable of specifically measuring activated IgM-free AIM without interference from IgM-bound AIM in blood. Although an enzyme-linked immunosorbent assay (ELISA) was used for IgM-free AIM [11, 19], a higherthroughput method is required considering large-scale utilization in clinical settings. To this end, we developed an antibody specific to IgM-free AIM and established a fully automatic and high-throughput electrochemiluminescence immunoassay (ECLIA) method that can specifically measure IgM-free AIM. Using this method, we examined the utility of IgM-free AIM as a diagnostic biomarker for HCC in each group of etiologies causing HCC by analyzing serum samples collected from patients with NASH, hepatitis B virus (HBV), and hepatitis $\mathrm{C}$ virus (HCV). Furthermore, we established the ECLIA method capable of measuring both IgM-free AIM and IgM-bound AIM (total AIM); we also investigated the existing AIM form in blood by measuring the fractionated samples from the sera of patients with NASH and NASH-HCC using size-exclusion chromatography to investigate the clinical significance of IgM-free AIM in NASH-HCC.

\section{Materials and methods}

\section{Subjects}

In total, 212 patients with liver disease, 117 without malignancy, and 95 with HCC who visited Saiseikai Suita Hospital from January 2010 to July 2017 were included in the study (Table 1). The study protocol was approved by the Human Ethics Committee of Saiseikai Suita Hospital. Informed consent was obtained from all patients, and the study protocol was performed in accordance with the Declaration of Helsinki. The serum samples of cancers other than liver cancer were provided by the Tsukuba Medical Laboratory of Education and Research Center and University of Tsukuba Hospital in Japan in accordance with the "Guidelines for clinical measurement and diagnosis technology improvement project," promoted by the same institutes. In addition to these specimens, serum samples were obtained from healthy volunteers who were employees of Sekisui Medical Co. (Tokyo, Japan). Written informed consent was obtained from all volunteers at the time of enrollment, in accordance with the code of ethics of Sekisui Medical Co. 
Table 1 List of specimens used in this study

Non-HCC (hepatitis and cirrhosis)

HCC tumor stage

$\begin{array}{llll}\mathrm{T} 1 & \mathrm{~T} 2 & \mathrm{~T} 3 & \mathrm{~T} 4\end{array}$

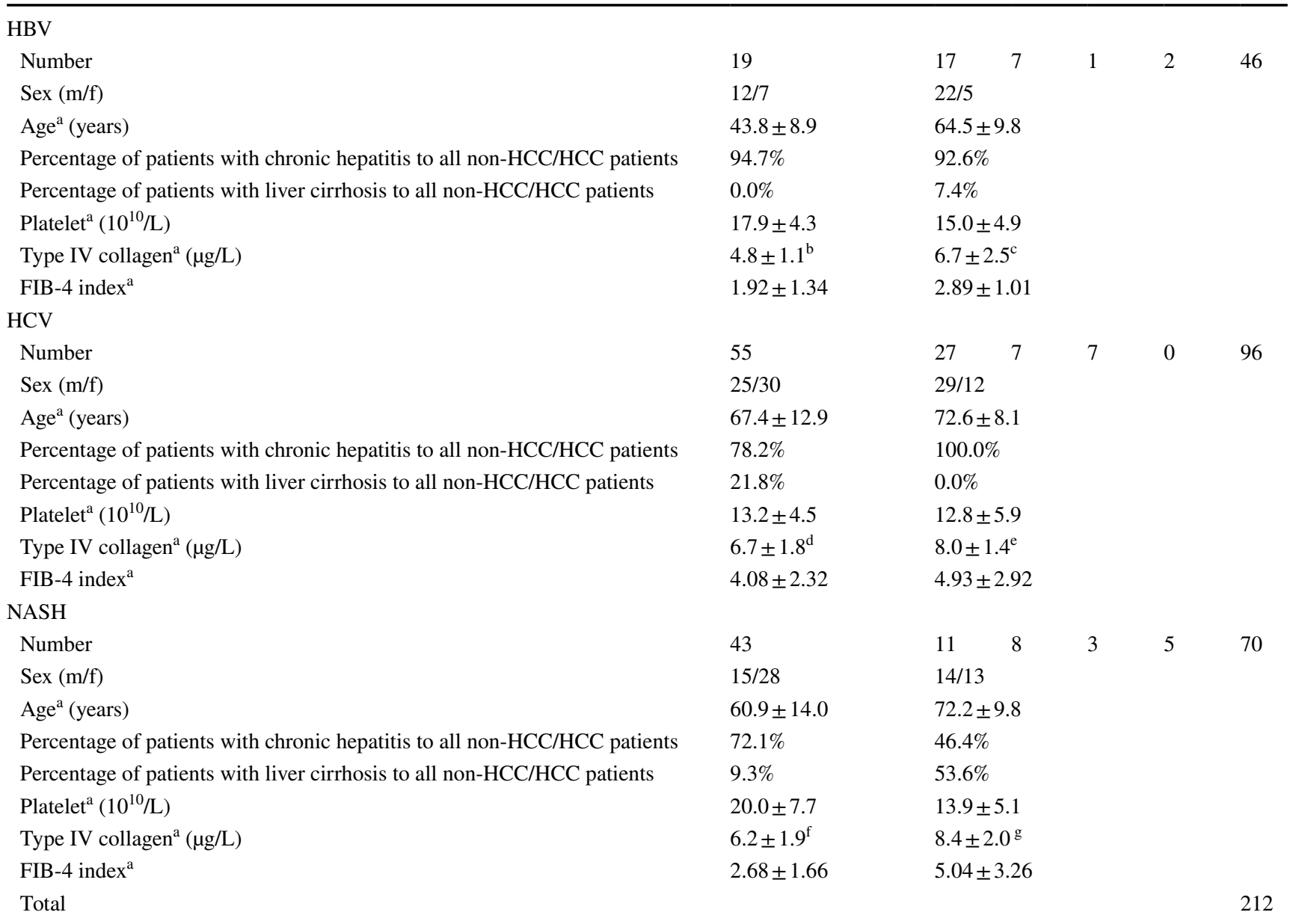

$H B V$ hepatitis B virus, $H C C$ hepatocellular carcinoma, $H C V$ hepatitis $\mathrm{C}$ virus, $N A S H$ non-alcoholic steatohepatitis

${ }^{a}$ Age, Platelet, Type IV collagen, and FIB-4 index are shown as mean \pm standard deviation

${ }^{b}$ Value for 18 patients (data of 1 patient that is missing was not considered)

${ }^{\mathrm{c}}$ Value for 25 patients (data of 2 patients that are missing were not considered)

${ }^{\mathrm{d}}$ Value for 54 patients (data of 1 patient that is missing was not considered)

${ }^{\mathrm{e}}$ Value for 36 patients (data of 5 patients that are missing were not considered)

${ }^{\mathrm{f}}$ Value for 42 patients (data of 1 patient is missing was not considered)

${ }^{\mathrm{g}}$ Value for 24 patients (data of 3 patients that are missing were not considered)

\section{Clinical and laboratory assessment}

Blood samples were obtained from the subjects in the morning after an overnight fast within 2 weeks prior to the liver biopsy and before each blood test. Clinical laboratory tests were conducted at the Department of Clinical Laboratory at Saiseikai Suita Hospital.

\section{Histopathological examination}

Liver biopsies were performed with a 16-gauge aspiration needle (Hakko Co., Ltd., Nagano, Japan), yielding specimens of at least $2.0 \mathrm{~cm}$ in length. The specimens were fixed in formalin, embedded in paraffin, and subjected to hematoxylin-eosin, Masson's trichrome, and Perl's iron 
staining. Histological assessment was performed by an expert hepatologist. Patients with NAFLD were classified into four types according to Matteoni's classification [5]: type 1, simple steatosis; type 2, steatosis with lobular inflammation; type 3 , type 2 plus ballooned hepatocytes; and type 4, presence of either Mallory-Denk bodies or fibrosis. Types 1 and 2 were classified as NAFL, and types 3 and 4 were classified as NASH. Furthermore, patients with NAFLD and fibrosis but without ballooning hepatocytes were classified as type 4. HCV infection was diagnosed based on the detection of HCV RNA using the Cobas 6800/8800 systems (Roche Molecular Systems Inc., CA, USA). HBV infection was diagnosed using an automated immunoassay analyzer LUMIPULSE (Fujirebio, Inc., Tokyo, Japan). HCC was diagnosed by histological examination or findings from ultrasound sonography, computed tomography (CT), magnetic resonance imaging (MRI), or hepatic angiography. Vascular invasion was assessed using dynamic CT, MRI, or angiography. DCP and AFP levels were measured in all patients. We used the optimal cut-off points of DCP and AFP in the analysis of $\mathrm{HCC}$ according to the manufacturer's instructions.

\section{Size-exclusion chromatography}

Size-exclusion chromatography of serum samples was conducted using a Prominence high-performance liquid chromatograph (HPLC) (Shimadzu Co., Ltd., Tokyo, Japan) with a G3000SW $\mathrm{XL}_{\mathrm{L}}$ column (Tosoh Co., Tokyo, Japan) at a flow rate of $1.0 \mathrm{~mL} / \mathrm{min}$ with $50 \mathrm{mM}$ phosphate-buffered saline ( $\mathrm{pH}$ 7.4). The fractionated samples were collected in $500 \mu \mathrm{L}$ aliquots.

\section{Preparation of anti-AIM monoclonal antibody}

To obtain anti-AIM monoclonal antibodies, BALB/c mice were immunized three times using full-length recombinant AIM with an adjuvant. Then, mouse spleen cells and myeloma cells were fused as previously described [20]. Antibody-producing clones that reacted with recombinant AIM and IgM-free AIM in the serum samples from patients with NASH-HCC were screened using ELISA and western blotting, followed by cloning using the limiting dilution method. The established clonal cells were injected into the abdominal cavity of BALB/cAJcL-nu/nu nude mice, and the $\mathrm{IgG}$ fraction was purified from the ascites fluid. One clonal cell line (No. 12) that produced antibodies specific to IgM-free AIM and two clonal cell lines (No. 8 and 11) that reacted with both IgM-free and IgM-bound AIM were selected with the ECLIA method described below.

\section{Preparation of ECLIA reagents}

Anti-AIM antibody No. 12 or 11 was mixed with magnetic beads with epoxy groups and incubated at $25{ }^{\circ} \mathrm{C}$ for 24-72 $\mathrm{h}$ to coat the beads with antibodies. The beads were then washed and blocked with Tris buffer ( $\mathrm{pH} 7.8$ ) containing $0.1 \% \mathrm{BSA}$ at $25{ }^{\circ} \mathrm{C}$ for $24 \mathrm{~h}$. Finally, the beads were washed with blocking buffer and stored at $4{ }^{\circ} \mathrm{C}$. Ruthenium $(\mathrm{Ru})$-labeled anti-AIM antibody No. 8 or 11 was prepared as follows. First, $\mathrm{Ru}$ with $\mathrm{N}$-hydroxysuccinimide residue (Origin Tag-NHS ESTER, IGEN, USA) was added to the anti-AIM antibody No. 8 or 11 solution and incubated at $25^{\circ} \mathrm{C}$ for $30 \mathrm{~min}$. Then, $2 \mathrm{M}$ glycine solution was added and incubated for $20 \mathrm{~min}$ to stop the reaction. Ru-labeled anti-AIM antibody No. 8 or 11 was subjected to gel filtration column chromatography (Sephadex G-25, GE Healthcare, Inc., USA) to remove unlabeled $\mathrm{Ru}$.

\section{Measurement of IgM-free AIM, IgM-bound AIM, and IgM using ECLIA}

IgM-free AIM, IgM-bound AIM, and IgM were measured using the ECLIA on a Picolumi III automatic analyzer (Sekisui Medical Co., Japan). Briefly, for measuring IgM-free AIM, $5 \mu \mathrm{L}$ serum sample was diluted with $100 \mu \mathrm{L}$ reaction buffer containing mouse $\operatorname{IgG}(200 \mu \mathrm{g} / \mathrm{mL})$; the diluted sample was then incubated with $25 \mu \mathrm{L}$ magnetic beads coated with anti-AIM antibody No. 12 at $30^{\circ} \mathrm{C}$ for 8 min (first reaction). When the HPLC fractionated sample was measured, $100 \mu \mathrm{L}$ undiluted fraction was used. After the first reaction, the beads were washed, and $100 \mu \mathrm{L} \mathrm{Ru}$-labeled anti-AIM antibody No. 11 was added and the mixture was incubated at $30^{\circ} \mathrm{C}$ for $8 \mathrm{~min}$ (second reaction).

The beads were then washed to remove the unreacted Ru-labeled antibody to form an electrode along with the electrolyte solution; the photons emitted from ruthenium by the electrochemical reaction were measured using a photomultiplier. The concentration of IgM-free AIM $(\mu \mathrm{g} / \mathrm{mL})$ was calculated based on the calibration curve from the photon count of the AIM standard concentrations $(0.1,1,4$, and $8 \mu \mathrm{g} / \mathrm{mL}$ ). The AIM standard was obtained by affinity purification from the supernatant of HEK293 cells expressing full-length recombinant AIM, using a Sepharose column immobilized with the anti-AIM antibody No. 12. The purified recombinant AIM was freeze-dried and weighed, and the concentration of each standard was determined based on the weight of the freeze-dried sample. When measuring both IgM-free AIM and IgM-bound AIM, magnetic beads coated with both anti-AIM antibody No. 11 and Ru-labeled anti-AIM antibody No. 8 were used for ECLIA as described above. To measure IgM, magnetic beads coated with commercially available anti-IgM monoclonal antibody (ADTEC 
Co., Japan) and the same antibody labeled with Ru were used for ECLIA.

\section{Receiver operating characteristic (ROC) curves for assessing the diagnostic accuracy of AIM}

ROC curves were obtained by calculating the sensitivity and specificity of the assay at every possible cut-off point and plotting sensitivity against [1-specificity] in SPSS for Windows Version 24 (SPSS Japan, Tokyo, Japan). The area under the ROC curve (AUROC) was calculated to determine the diagnostic accuracy of the assay. Appropriate cut-off points were examined for balancing the sensitivity and specificity of the ROC curve, and the optimal cut-off point was identified as that yielding the minimal value for $\left[(1-\text { sensitivity })^{2}+(1-\text { specificity })^{2}\right]$ or the maximal value for [sensitivity + specificity -1$][21]$.

\section{Statistical analyses}

Statistical analysis was performed using IBM SPSS Statistics Version 24 for Windows. All values are expressed as the mean \pm standard deviation. Differences in mean values between groups were assessed using the Mann-Whitney U-test, whereas correlations between parameters were evaluated using Pearson's correlation coefficient. For all analyses, statistical significance was set at $p<0.05$.

\section{Results}

\section{Analytical performance of the newly established ECLIA specific for IgM-free AIM}

\section{Evaluation of specificity for IgM-free AIM}

To evaluate the analytical performance of ECLIA, sizeexclusion chromatography fractionated samples from the serum of one patient with NASH-HCC were assayed by the ECLIA using magnetic beads coated with anti-AIM antibody No. 11 and Ru-labeled anti-AIM antibody No. 8 to measure IgM-free AIM and IgM-bound AIM. Bimodal peaks of ECLIA counts were observed in fractions No. 4-6 and No. 12-13 (Fig. 1a). In contrast, for IgM-free AIMspecific ECLIA using beads coated with anti-AIM antibody No. 12 and Ru-labeled anti-AIM antibody No. 11, only one peak of ECLIA counts was observed in fractions No. 12-13 (Fig. 1b). Furthermore, using ECLIA to measure IgM, only one peak of ECLIA counts was observed in fractions No. 4-6 (Fig. 1c). These data indicate that IgM-bound AIM and IgM-free AIM were fractionated in fractions No. 4-6 and No. 12-13, respectively, and that ECLIA using beads coated with anti-AIM antibody No. 12 and Ru-labeled anti-AIM antibody No. 11 specifically measures IgM-free AIM, but not IgM-bound AIM.

Next, 25 serum samples from healthy individuals $(n=5)$ and patients with NAFL $(n=5)$, NASH $(n=5)$, and NASHHCC $(n=10)$ were fractionated by size-exclusion chromatography, and both IgM-bound AIM and IgM-free AIM were measured by ECLIA using beads coated with anti-AIM antibody No. 11 and Ru-labeled anti-AIM antibody No. 8. Furthermore, the same serum samples were directly measured
Fig. 1 ECLIA counts of sizeexclusion chromatography fractionated samples from the serum of one patient with NASH-HCC. The results of ECLIA for a IgM-free AIM and IgM-bound AIM, b IgMfree AIM, and $\mathbf{c}$ IgM alone. a Bimodal peaks of ECLIA counts observed in fractions No. 4-6 and 12-13. b One peak of ECLIA counts observed in fractions No. 12-13. $\mathbf{c}$ One peak of ECLIA counts observed in fractions No. 4-6. AIM apoptosis inhibitor of macrophage, ECLIA electrochemiluminescence immunoassay, $H C C$ hepatocellular carcinoma, $\mathrm{NASH}$ nonalcoholic steatohepatitis a

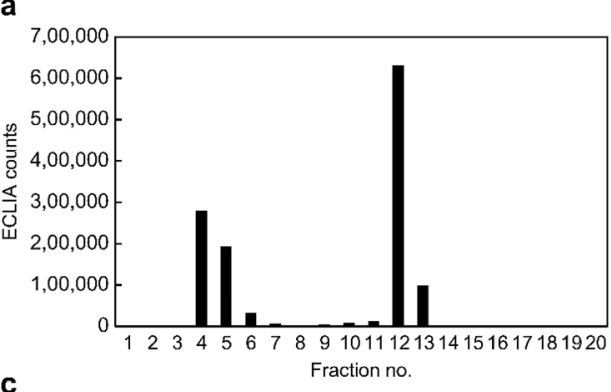

c

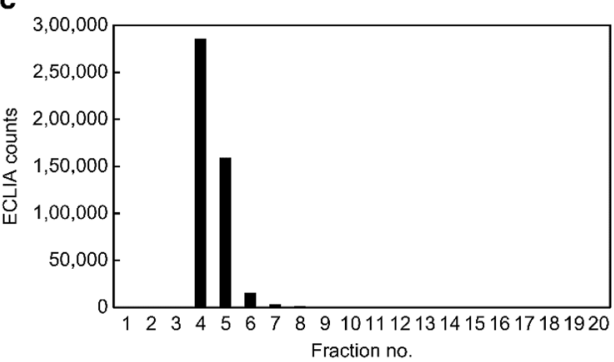

b

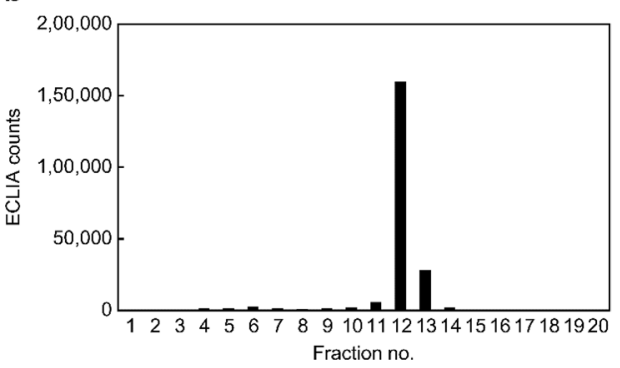


for IgM-free AIM concentration by ECLIA using anti-AIM antibody No. 12 and Ru-labeled anti-AIM antibody No. 11 to investigate its correlation with the fractionated IgM-free AIM samples by size-exclusion chromatography. The sum of ECLIA counts from fraction No. 12-13 by size-exclusion chromatography of the 25 serum samples and directly measured IgM-free AIM concentration were well-correlated ( $r=0.98, p<0.0001$; Online Resource 1), indicating that ECLIA using anti-AIM antibody No. 12 and Ru-labeled antiAIM antibody No. 11 specifically and accurately measures the IgM-free AIM concentration in serum samples.

\section{Evaluation of accuracy for IgM-free AIM measurement}

To evaluate the sample dilution linearity, two serum samples from patients with NASH-HCC (No. 1 and 2) were serially diluted from 1:1 to 1:64 and IgM-free AIM was measured by ECLIA using beads coated with anti-AIM antibody No. 12 and Ru-labeled anti-AIM antibody No. 11. Good linearity was observed for each serum sample (Online Resource $2 a$ and $b$ ).

Next, assay repeatability was evaluated by five consecutive assays of IgM-free AIM in three serum samples, and the intra-assay coefficients of variation were $0.5-4.0 \%$. Furthermore, assay reproducibility was evaluated by assaying three serum samples on four different days, and the interday assay coefficients of variation were 1.8-3.2\%. Both the intra-assay and the inter-day assay coefficients of variation obtained indicated good precision.
Finally, the effect of interfering substances was examined by spiking the serum samples with Interference Check A Plus and RF Plus (Sysmex, Japan) and measuring the IgMfree AIM using ECLIA. No change in the IgM-free AIM level was observed in serum samples spiked with bilirubin $\mathrm{F}$ and $\mathrm{C}$ up to $25 \mathrm{mg} / \mathrm{dL}$, hemoglobin up to $500 \mathrm{mg} / \mathrm{dL}$, chyle up to $3,000 \mathrm{FTU}$, and rheumatoid factor (RF) up to $500 \mathrm{IU} /$ $\mathrm{mL}$ (Online Resource 3), indicating that the assay was not affected by interfering substances.

\section{IgM-free AIM concentration in sera from patients with liver disease}

To evaluate the diagnostic value of IgM-free AIM, sera from the non-HCC (chronic hepatitis and cirrhosis) patient group and the HCC patient group including all cancer stages related to NASH, HCV, and HBV were measured with ECLIA using the anti-AIM antibody No. 12 and No. 11. The IgM-free AIM value was significantly higher in the HCC patient group than in the non-HCC patient group $(p<0.001$; Fig. 2a-d). The same result was obtained when patients with HCC were limited to stages 1-2 (Online Resource 4). Furthermore, we compared the ROC curves of IgM-free AIM calculated from specificity and sensitivity with those of the conventional HCC biomarkers, AFP and DCP, for each liver disease related to NASH, HCV, HBV, and for all the patients. IgM-free AIM showed the highest AUROC in each disease group including all cancer stages of HCC and in all the patients together (Fig. 3a-d); the same result was obtained when HCC was limited to stages 1-2 (Online Resource 5).
Fig. 2 Distribution mapping of serum IgM-free AIM level in non-HCC (hepatitis and cirrhosis) and HCC patient groups for all cancer stages. The results are shown in $\mathbf{a}-\mathbf{d}$ are for all cancer stages of NASH-HCC, HBV-HCC, HCV-HCC, and all the patients, respectively. The serum IgM-free AIM level was significantly higher in the HCC patient group than that in the non-HCC patient group $(p<0.001)$ regardless of the pathogenesis. $* p<0.001$ (Mann-Whitney U-test). AIM apoptosis inhibitor of macrophage, $H B V$ hepatitis B virus, $H C C$ hepatocellular carcinoma, $\mathrm{HCV}$ hepatitis $\mathrm{C}$ virus, $\mathrm{NASH}$ non-alcoholic steatohepatitis
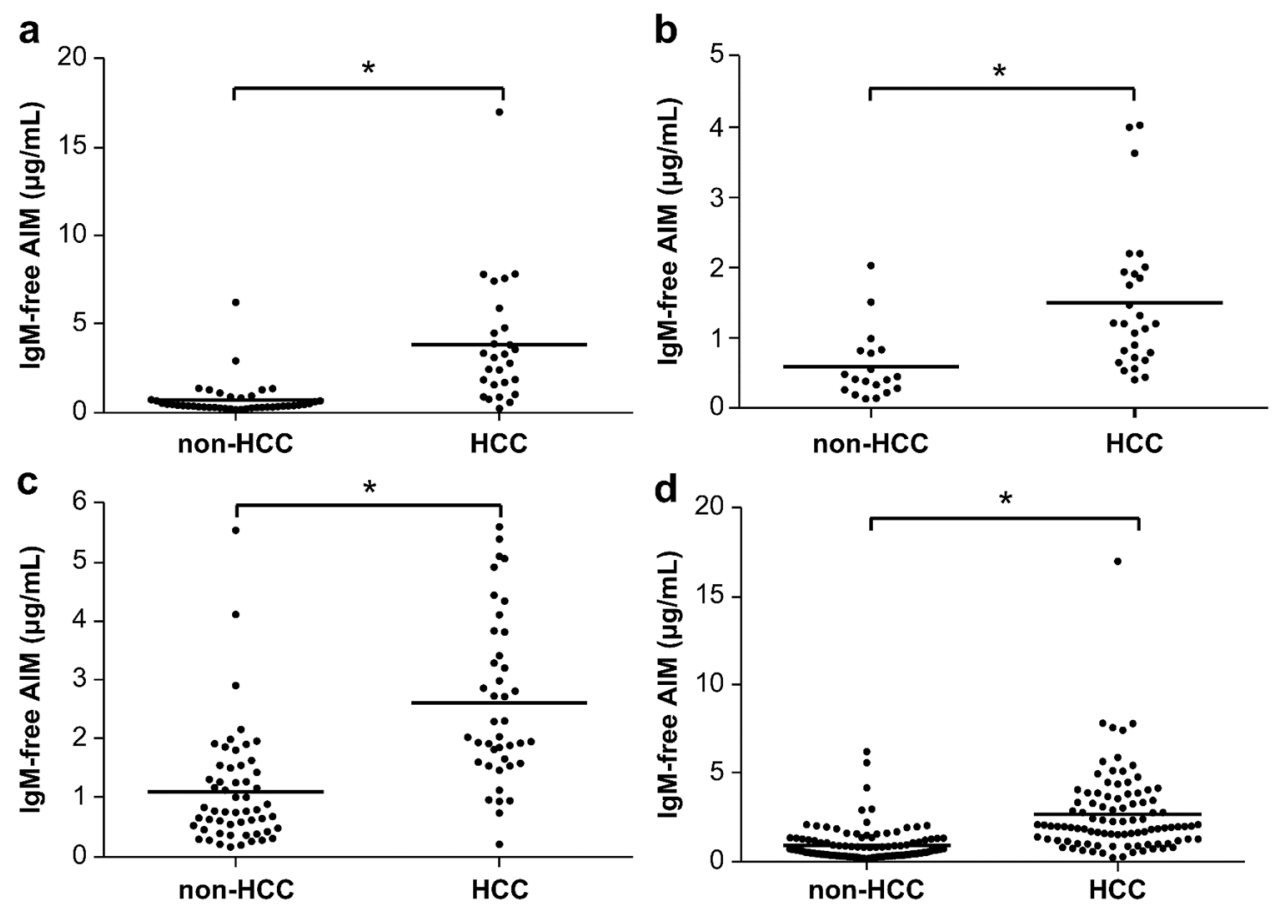
Fig. 3 ROC analyses of IgMfree AIM, AFP, and DCP for all cancer stages. The sensitivity and specificity of the markers were determined to discriminate the patients with HCC from those without HCC a-d Include all cancer stages of NASH-HCC, HBV-HCC, HCV-HCC, and all the patients, respectively. IgM-free AIM showed the highest AUROC for each disease group and all the patients. $A F P$ alpha-fetoprotein, $A I M$ apoptosis inhibitor of macrophage, $D C P$ des- $\gamma$-carboxy prothrombin, $H B V$ hepatitis $\mathrm{B}$ virus, $H C C$ hepatocellular carcinoma, $H C V$ hepatitis $\mathrm{C}$ virus, $N A S H$ non-alcoholic steatohepatitis, $R O C$ receiver operating characteristic, $A U R O C$ area under the receiver operating characteristic

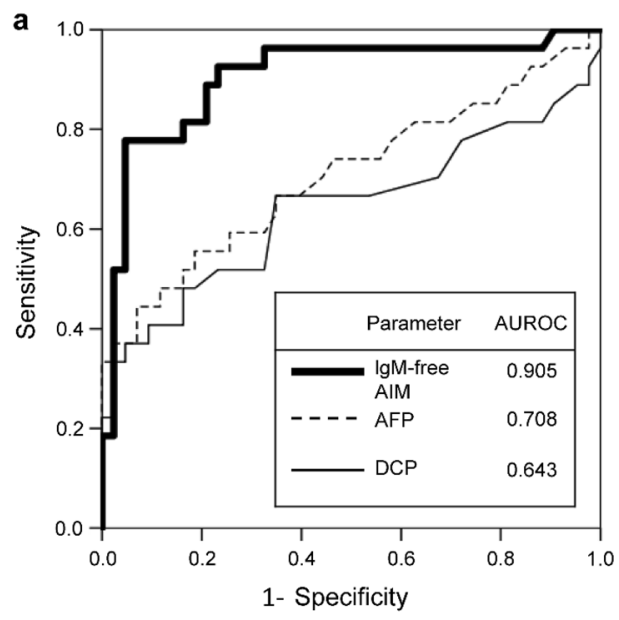

c

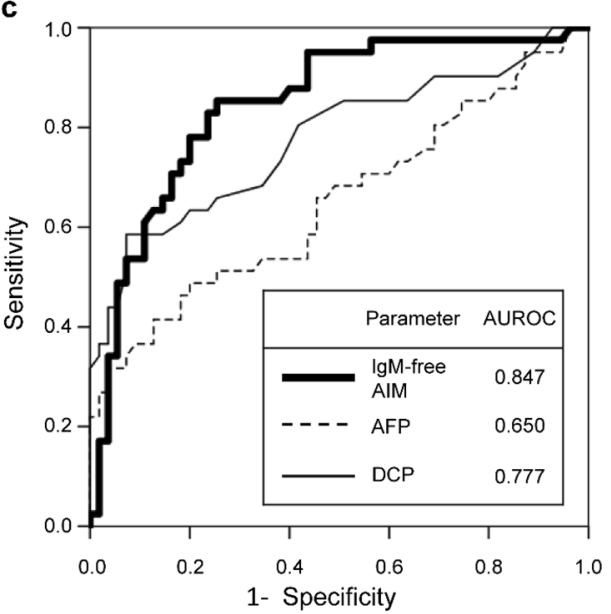

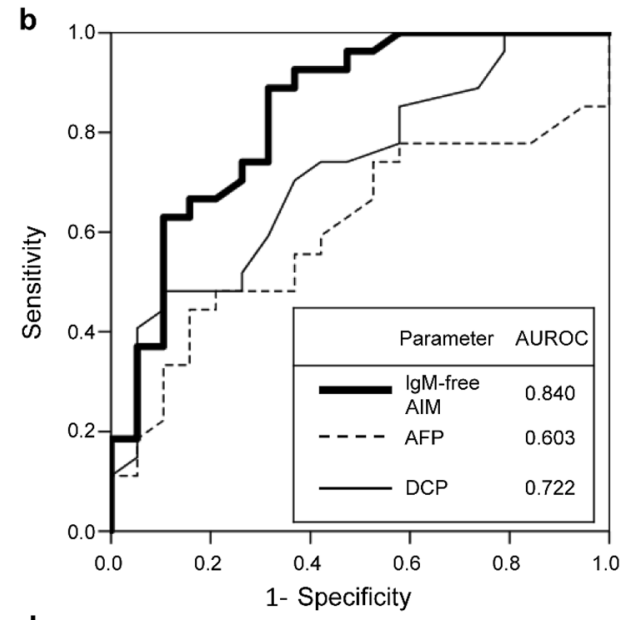

d

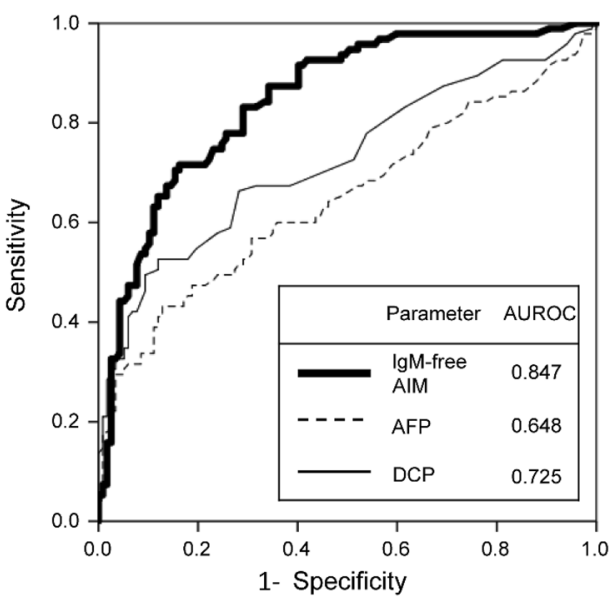

Table 2 Comparison of the sensitivity, specificity, and accuracy of IgM-free AIM, AFP, and DCP for discriminating $\mathrm{HCC}$ of all cancer stages

\begin{tabular}{|c|c|c|c|c|}
\hline & Marker & Sensitivity (\%) & Specificity (\%) & Accuracy $(\%)$ \\
\hline \multicolumn{5}{|c|}{ Comparison of diagnostic performance for NASH-HCC } \\
\hline \multirow[t]{3}{*}{ All cancer stages } & IgM-free AIM & 74.1 & 95.3 & 87.1 \\
\hline & AFP & 18.5 & 100 & 68.6 \\
\hline & DCP & 40.7 & 90.7 & 71.4 \\
\hline \multicolumn{5}{|c|}{ Comparison of diagnostic performance for HBV-HCC } \\
\hline \multirow[t]{3}{*}{ All cancer stages } & IgM-free AIM & 37.0 & 94.7 & 60.9 \\
\hline & AFP & 33.3 & 84.2 & 54.3 \\
\hline & DCP & 33.3 & 94.7 & 58.7 \\
\hline \multicolumn{5}{|c|}{ Comparison of diagnostic performance for HCV-HCC } \\
\hline \multirow[t]{3}{*}{ All cancer stages } & IgM-free AIM & 87.8 & 80.0 & 83.3 \\
\hline & AFP & 41.5 & 87.3 & 67.7 \\
\hline & DCP & 41.5 & 96.4 & 72.9 \\
\hline \multicolumn{5}{|c|}{ Comparison of diagnostic performance for all $\mathrm{HCC}$ patients } \\
\hline \multirow[t]{3}{*}{ All cancer stages } & IgM-free AIM & 69.5 & 88.0 & 79.7 \\
\hline & AFP & 32.6 & 91.5 & 65.1 \\
\hline & DCP & 38.9 & 94.0 & 69.3 \\
\hline
\end{tabular}

The cut-off values of IgM-free AIM, AFP, and DCP were set at $1.6 \mu \mathrm{g} / \mathrm{mL}, 20 \mathrm{ng} / \mathrm{mL}$, and $40 \mathrm{mAU} / \mathrm{mL}$, respectively

$A F P$ alpha-fetoprotein, AIM apoptosis inhibitor of macrophage, $D C P$ des- $\gamma$-carboxy prothrombin, $H B V$ hepatitis B virus, $H C C$ hepatocellular carcinoma, $H C V$ hepatitis $\mathrm{C}$ virus, $N A S H$ non-alcoholic steatohepatitis 
After balancing the sensitivity and specificity of the ROC curve calculated from all non-HCC groups $(n=117)$ and all HCC groups $(n=95)$, the optimal cut-off of serum IgMfree AIM for predicting HCC was estimated to be $1.6 \mu \mathrm{g} /$ $\mathrm{mL}$ as it showed the maximal diagnostic accuracy. Using this cut-off point, the sensitivity, specificity, and accuracy of IgM-free AIM were determined and compared with those of AFP and DCP, which were set to $20 \mathrm{ng} / \mathrm{mL}$ and $40 \mathrm{mAU} /$ $\mathrm{mL}$, respectively. The accuracy of IgM-free AIM for HCC detection related to NASH, HBV, and HCV was $87.1 \%$, $60.9 \%$, and $83.3 \%$, respectively, and that in all the patients was $79.7 \%$, indicating that IgM-free AIM has a higher clinical accuracy than AFP or DCP (Table 2). The same result was obtained when HCC was limited to stages 1-2 (Online Resource 6). IgM-free AIM is produced by macrophages, whereas DCP and AFP are produced by liver cancer cells $[22,23]$; therefore, a combination assay of IgM-free AIM with AFP or DCP may be useful for HCC diagnosis. We then investigated the correlation between IgM-free AIM and AFP or DCP. IgM-free AIM did not show a definite correlation with AFP $(r=0.224, p=0.001$; Online Resource 7a) or DCP $(r=0.139, p=0.043$; Online Resource 7b). Furthermore, due to its higher positivity rate, IgM-free AIM alone can be a more sensitive marker for HCC than AFP or DCP alone, respectively (63.2\% vs $33.7-38.9 \%$; Online Resource $8 \mathrm{a}-\mathrm{c})$. In addition, we compared the positivity rate in the combination of IgM-free AIM and AFP or DCP with that in the combination of AFP and DCP. The positivity rates in the combination of IgM-free AIM and AFP, and that of IgM-free AIM and DCP were $74.7 \%$ and $77.9 \%$, respectively, indicating that the positivity rates of IgM-free AIM in combination with AFP or DCP are higher than those in the combination of AFP and DCP (49.5\%; Online Resource $8 \mathrm{~d}-\mathrm{f}$ ). It is notable that IgM-free AIM alone showed a higher sensitivity than the combination of AFP and DCP.

\section{IgM-free AIM levels in cancers other than liver cancer, and the relationship of IgM-free AIM between cancer stage and age}

To explore whether IgM-free AIM is elevated specifically in patients with liver cancer, we also measured IgM-free AIM levels in patients with different types of cancer. IgMfree AIM was significantly high only in patients with liver cancer and was not elevated in patients with other types of cancer compared to normal volunteers (Online Resource 9). On the other hand, there was no difference in IgM-free AIM levels between patients with different cancer stages (Online Resource 10), and no correlation was seen between IgMfree AIM and age regardless of the presence of HCC and non-HCC (Online Resource 11), indicating that the stage of cancer and the age are independent of IgM-free AIM level.
Measurement of IgM-free and IgM-bound AIM in fractionated serum samples by size-exclusion chromatography

Considering that patients with HCC showed higher positivity for IgM-free AIM in their sera, we investigated the existing form of AIM in blood. Therefore, the sera from healthy volunteers and patients with NAFL, NASH, and NASH-HCC were fractionated by size-exclusion chromatography, and the fractionated samples were assayed for IgM-bound AIM and IgM-free AIM by ECLIA using beads coated with antiAIM antibody No. 11 and Ru-labeled anti-AIM antibody No. 8. AIM antigen activity was observed in fractions No. 4-6 (IgM-bound AIM) and fractions No. 12-13 (IgM-free AIM) from all patients (Online Resource 12). Interestingly, the peaks of fractions No. 4-6 (IgM-bound AIM) from healthy volunteers and patients with NAFL and NASH were higher than the peaks of fractions No. 12-13 (IgM-free AIM), whereas the peaks of fractions No. 12-13 (IgM-free AIM) from patients with NASH-HCC were higher than the peaks of fractions No. 4-6 (IgM-bound AIM). Subsequently, 25 serum samples from healthy volunteers $(n=5)$ and patients with NAFL $(n=5)$, NASH $(n=5)$, and NASH-HCC $(n=10)$ were fractionated in the same manner and assayed for IgMbound AIM, IgM-free AIM, and IgM by ECLIA. The IgMfree AIM counts and IgM-free/IgM-bound AIM count ratios were significantly higher in the NASH-HCC group than in the other groups $(p<0.001$; Fig. $4 \mathrm{a}, \mathrm{b})$. The IgM-bound AIM counts of patients with NASH-HCC did not significantly increase compared with those of healthy volunteers and patients with NASH; however, a weak, yet statistically significant, increase was observed compared with the NAFL group ( $p=0.028$; Fig. $4 c)$. The IgM counts of patients with NASH-HCC were not significantly higher than those of patients in the other groups ( $p=0.032$; Fig. $4 d$ ). Furthermore, we evaluated the correlations of ECLIA counts among IgM-free AIM, IgM-bound AIM, and IgM in all serum samples. IgM-bound AIM correlated well with $\operatorname{IgM}(r=0.78$, $p<0.0001)$, whereas IgM-free-AIM did not $(r=0.110$, $p=0.601 ;$ Fig. $5 \mathrm{a}, \mathrm{b})$. There was a weak positive correlation between IgM-bound AIM and IgM-free-AIM $(r=0.572$, $p=0.003$; Fig. $5 \mathrm{c}$ ). These data indicate that IgM-free AIM counts are specifically increased in the blood of patients with NASH-HCC because of dissociation from IgM.

\section{Discussion}

AIM plays a key role in protecting liver cells from lipid accumulation and carcinogenesis, and the release of free AIM from IgM is essential for exerting its biological function [11, 17, 24]. Therefore, IgM-free AIM is considered a candidate diagnostic marker for liver diseases. Moreover, 

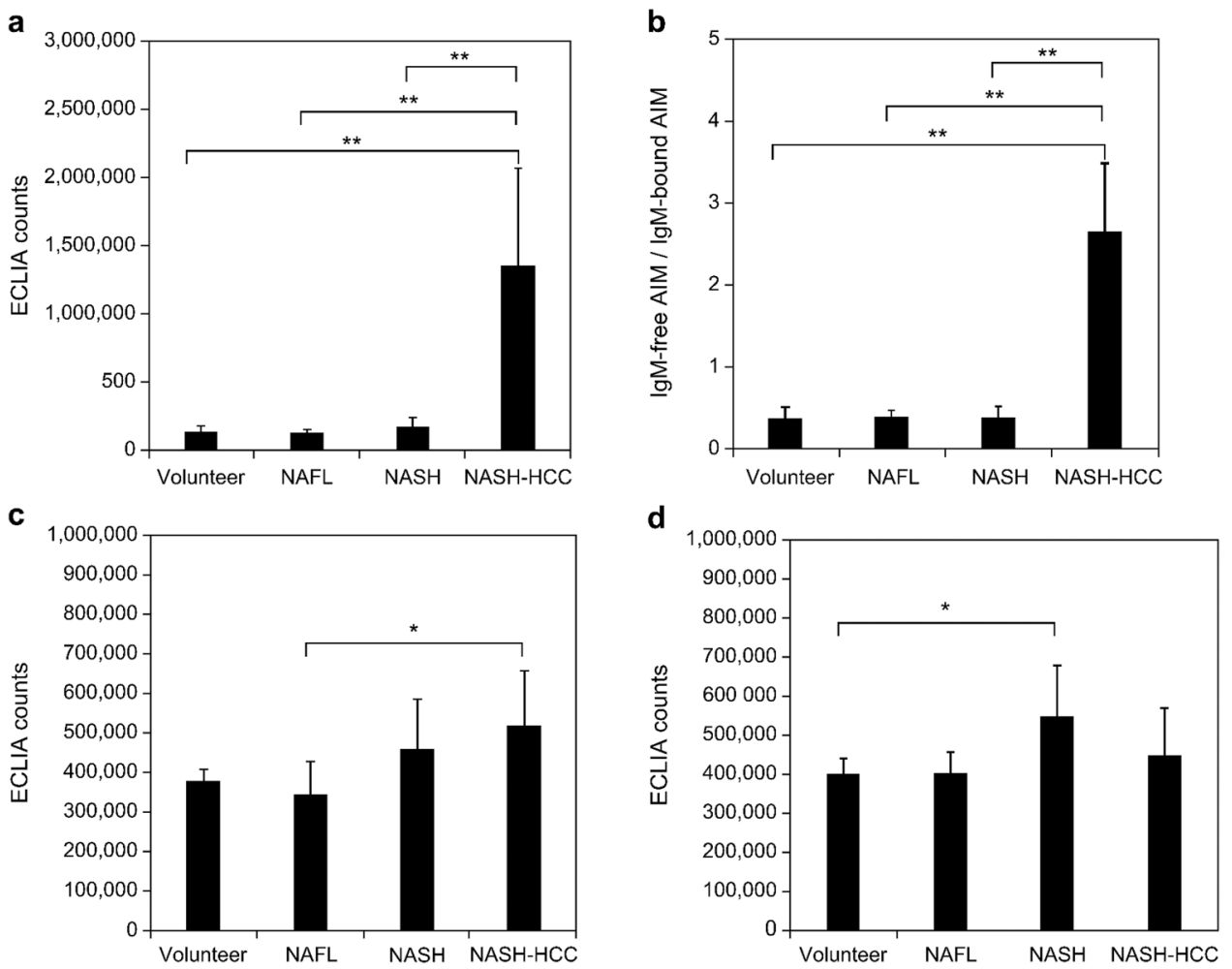

Fig. 4 ECLIA counts of size-exclusion chromatography fractionated serum samples from healthy volunteers and from patients with NAFL, NASH, and NASH-HCC. The mean values of ECLIA counts for a IgM-free AIM, b IgM-free AIM/IgM-bound AIM ratio, c IgMbound AIM, and $\mathbf{d}$ IgM determined from the fractionated samples in each disease ( 25 serum samples from healthy volunteers $(n=5)$, and from patients with NAFL $(n=5)$, NASH $(n=5)$, and NASH-HCC

$(n=10))$. a IgM-free and b IgM-free AIM/IgM-bound AIM ratio were significantly higher in patients with NASH-HCC. c IgM-bound AIM and $\mathbf{d} \operatorname{IgM}$ were relatively higher in patients with NASH and NASH-HCC. $* * p<0.001, * p<0.05$ (Mann-Whitney U-test). AIM apoptosis inhibitor of macrophage, ECLIA electrochemiluminescence immunoassay, $H C C$ hepatocellular carcinoma, NAFL non-alcoholic fatty liver, $N A S H$ non-alcoholic steatohepatitis
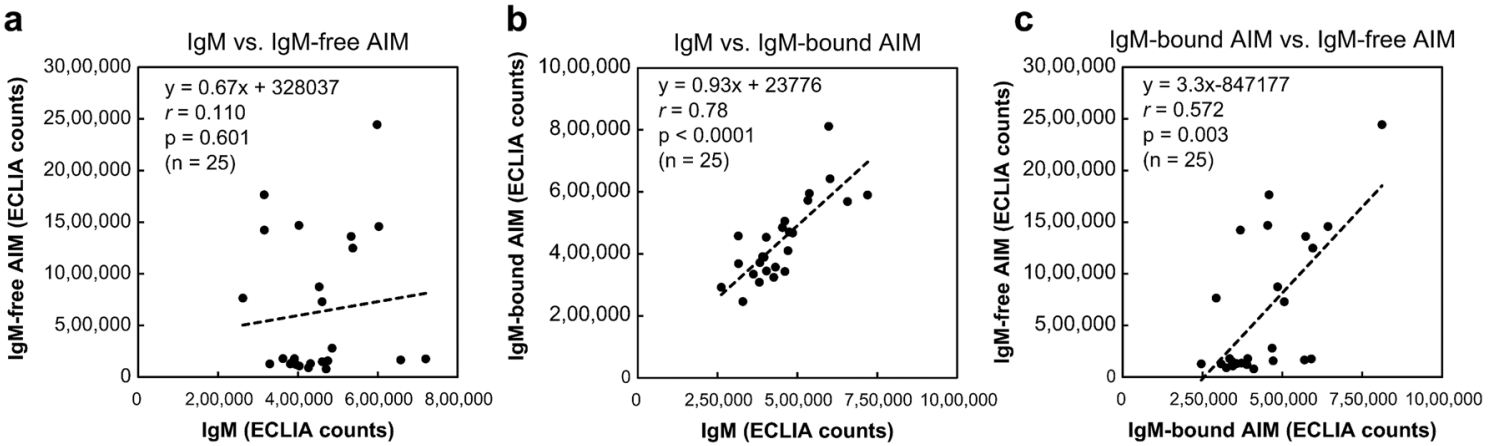

Fig. 5 Correlations of ECLIA counts among IgM, IgM-bound AIM, and IgM-free AIM fractions. a No significant correlation was observed between the ECLIA counts of IgM and IgM-free AIM. b ECLIA counts of IgM and IgM-bound AIM showed a significant pos-

serum IgM-free AIM levels are a sensitive diagnostic marker for NASH-HCC [11].

In this study, we established a novel high-throughput method, ECLIA, that enables us to automatically measure itive correlation. c ECLIA counts of IgM-bound and IgM-free AIM showed a weak positive correlation. AIM apoptosis inhibitor of macrophage, ECLIA electrochemiluminescence immunoassay

the IgM-free AIM levels, and verified its specificity for IgM-free AIM. It specifically and precisely measured IgMfree AIM without reacting with IgM-bound AIM in serum samples. 
Serum IgM-free AIM levels were significantly higher in both the NASH-HCC patient group and the viral HCC patient group, and both ROC analysis and the diagnostic accuracy of IgM-free AIM demonstrate that serum IgM-free AIM may be a potential universal HCC diagnostic marker that could be superior to AFP or DCP, even in early cancer stages. In fact, a mouse carcinogenicity study indicated that AIM begins accumulating on the hepatic cell surface at the preneoplastic stage to exert an anti-HCC effect through complement activation [17]. Thus, serum IgM-free AIM levels may be elevated in the early stage of HCC, even in humans. This report may also explain why some non-HCC patients showed high IgM-free AIM levels in this study, i.e., AIM released from IgM may start to eliminate cancer cells in the very early stages of HCC which cannot be diagnosed by conventional tools. This result is consistent with the previous report [11]. In fact, considering the growth rate of HCC tumors reported in a previous study [25], it may take several years until HCC lesions with an approximate diameter of $1 \mathrm{~cm}$ can be detected using ultrasound or computed tomography; therefore, further follow-up studies are needed to demonstrate whether non-HCC patients showing high IgM-free AIM levels already have microcarcinoma. On the other hand, there was no difference in IgM-free AIM levels between patients with different cancer stages, which may also be related to the hypothesis that AIM is released from IgM in the early stages of HCC. Furthermore, according to this study, the combination of IgM-free AIM with AFP or DCP can be more useful for the diagnosis of HCC than that of AFP and DCP. Thought to be one of the immunological parameters produced by macrophages, AIM-which is different from AFP or DCP produced by cancer tissue-may be a unique biomarker for HCC. AIM is reported to be produced mainly by liver Kupffer cells [26], and this may be the reason why IgM-free AIM increases less in other cancer types than in liver cancer. However, further research is needed to elucidate why a higher IgM-free AIM level was observed in NASH-HCC than in HBV-HCC and HCV-HCC.

As patients with HCC showed higher IgM-free AIM positivity, we sought to investigate the existing form of AIM in the blood by size-exclusion chromatography, since AIM has been shown to be associated with the IgM pentamers in blood and this association protects AIM from renal excretion [27]. Consequently, the specific increase in IgM-free AIM levels in the serum of patients with NASHHCC was verified. Interestingly, IgM levels in these patients were significantly correlated with IgM-bound AIM levels; however, they did not correlate with IgM-free AIM levels, indicating that IgM-free AIM is a superior marker to IgM-bound AIM, as it is not influenced by $\operatorname{IgM}$ concentration, which can cause sex-based differences and might be influenced by liver function [27]. Although the increase in serum IgM-free AIM levels represents AIM dissociation in response to HCC occurrence, the precise mechanism underlying disease-specific dissociation remains unclear. Based on our structural analysis of the binding mode of AIM with IgM pentamer using an electron microscope [14], certain reactions that are induced upon HCC development might target the disulfide bond or the charge-based interaction between AIM and IgM Fc. However, further studies are required to elucidate the underlying mechanism.

In conclusion, we developed a clinically feasible ECLIA method for measuring IgM-free AIM in serum and demonstrated that IgM-free AIM is a more precise biomarker for HCC than the conventional biomarkers, AFP or DCP, for NASH-HCC, as well as for HBV- or HCV-related $\mathrm{HCC}$, even in early cancer stages. The ECLIA method is expected to contribute to further clinical studies on AIM, which has the potential to serve as a universal biomarker for in vitro HCC diagnosis. However, due to the limited number of samples from patients with HCC available for this study, particularly those from patients in an early cancer stage, further investigation is warranted to confirm the clinical significance of IgM-free AIM as a diagnostic marker for early HCC. In addition, further study is needed to examine whether IgM-free AIM also increases in the liver malignancies that were not evaluated in this study, such as cholangiocellular carcinoma, metastatic liver cancer, and so on.

Supplementary Information The online version contains supplementary material available at https://doi.org/10.1007/s12328-021-01567-4.

Acknowledgements This study was conducted based on a research collaboration contract between Saiseikai Suita Hospital and Sekisui Medical Co Ltd. and on a research collaboration contract between Toru Miyazaki at Tokyo University and Sekisui Medical Co Ltd. The authors thank the Department of Clinical Laboratory of Saiseikai Suita Hospital for providing suggestions and for conducting the clinical laboratory tests.

Author contributions TS: experiments and procedures, assembly of data, writing and critical revision of the article. TS: experiments and procedures, collection and assembly of data, writing of the article. TA: concept and design, assembly of data. YK: experiments and procedures, collection of data. JH: experiments and procedures. MM: interpretation of data. $\mathrm{TN}$ : interpretation of data. TM: concept and design, experiments and procedures. TO: concept and design, final approval of the article.

Funding No financial support to declare.

\section{Declarations}

Conflict of interest Employment: Tomo Shimizu/Sekisui Medical Co., Ltd. Takashi Sawada/Sekisui Medical Co., Ltd. Tomohide Asai/Sekisui Medical Co., Ltd. Yuka Kanetsuki/Sekisui Medical Co., Ltd. Jiro Hirota/Sekisui Medical Co., Ltd. 
Human/animal rights All procedures followed have been performed in accordance with the ethical standards laid down in the 1964 Declaration of Helsinki and its later amendments.

Informed consent Informed consent was obtained from all patients for being included in the study.

Open Access This article is licensed under a Creative Commons Attribution 4.0 International License, which permits use, sharing, adaptation, distribution and reproduction in any medium or format, as long as you give appropriate credit to the original author(s) and the source, provide a link to the Creative Commons licence, and indicate if changes were made. The images or other third party material in this article are included in the article's Creative Commons licence, unless indicated otherwise in a credit line to the material. If material is not included in the article's Creative Commons licence and your intended use is not permitted by statutory regulation or exceeds the permitted use, you will need to obtain permission directly from the copyright holder. To view a copy of this licence, visit http://creativecommons.org/licenses/by/4.0/.

\section{References}

1. Schwimmer JB, Deutsch R, Kahen T, et al. Prevalence of fatty liver in children and adolescents. Pediatrics. 2006;118:1388-93.

2. European Association for the Study of the Liver, European Association for the Study of Diabetes, European Association for the Study of Obesity. EASL-EASD-EASO Clinical Practice Guidelines for the management of non-alcoholic fatty liver disease. $J$ Hepatol. 2016;64:1388-402.

3. Chalasani N, Younossi Z, Lavine JE, et al. The diagnosis and management of nonalcoholic fatty liver disease: practice guidance from the American Association for the Study of Liver Diseases. Hepatology. 2018;67:328-57.

4. Okanoue T, Umemura A, Yasui K, et al. Nonalcoholic fatty liver disease and nonalcoholic steatohepatitis in Japan. J Gastroenterol Hepatol. 2011;26:153-62.

5. Matteoni CA, Younossi ZM, Gramlich T, et al. Nonalcoholic fatty liver disease: a spectrum of clinical and pathological severity. Gastroenterology. 1999;116:1413-9.

6. Zopf S, Kremer AE, Neurath MF, et al. Advances in hepatitis C therapy: what is the current state - what come's next? World J Hepatol. 2016;28:139-47.

7. Koda M, Nagahara T, Matono T, et al. Nucleotide analogs for patients with HBV-related hepatocellular carcinoma increase the survival rate through improved liver function. Intern Med. 2009;48:11-7.

8. Takuma Y, Nouso K. Nonalcoholic steatohepatitis-associated hepatocellular carcinoma: our case series and literature review. World J Gastroenterol. 2010;16:1436-41.

9. Bullock RE, Zaitoun AM, Aithal GP, et al. Association of nonalcoholic steatohepatitis without significant fibrosis with hepatocellular carcinoma. J Hepatol. 2004;41:685-6.

10. Yasui K, Hashimoto E, Komorizono Y, et al. Characteristics of patients with nonalcoholic steatohepatitis who develop hepatocellular carcinoma. Clin Gastroenterol Hepatol. 2011;9:428-33.

11. Koyama N, Yamazaki T, Kanetsuki Y, et al. Activation of apoptosis inhibitor of macrophage is a sensitive diagnostic marker for NASH-associated hepatocellular carcinoma. J Gastroenterol. 2018;53:770-9.

12. Miyazaki T, Hirokami Y, Matsuhashi N, et al. Increased susceptibility of thymocytes to apoptosis in mice lacking AIM, a novel murine macrophage-derived soluble factor belonging to the scavenger receptor cysteine-rich domain superfamily. J Exp Med. 1999;189:413-22.

13. Tissot JD, Sanchez JC, Vuadens F, et al. IgM are associated to Sp alpha (CD5 antigen-like). Electrophoresis. 2002;23:1203-6.

14. Hiramoto E, Tsutsumi A, Suzuki R, et al. The IgM pentamer is an asymmetric pentagon with an open groove that binds the AIM protein. Sci Adv. 2018;4:1199.

15. Miyazaki T, Yamazaki T, Sugisawa R, et al. AIM associated with the IgM pentamer: attackers on stand-by at aircraft carrier. Cell Mol Immunol. 2018;15:563-74.

16. Kurokawa J, Arai S, Nakashima K, et al. Macrophage-derived AIM is endocytosed into adipocytes and decreases lipid droplets via inhibition of fatty acid synthase activity. Cell Metab. 2010;11:479-92.

17. Maehara N, Arai S, Mori M, et al. Circulating AIM prevents hepatocellular carcinoma through complement activation. Cell Rep. 2014;9:61-74

18. Arai S, Kitada K, Yamazaki T, et al. Apoptosis inhibitor of macrophage protein enhances intraluminal debris clearance and ameliorates acute kidney injury in mice. Nat Med. 2016;22:183-93.

19. Mera K, Uto H, Mawatari S, et al. Serum levels of apoptosis inhibitor of macrophage are associated with hepatic fibrosis in patients with chronic hepatitis C. BMC Gastroenterol. 2014;14:27.

20. Köhler G, Milstein C. Continuous cultures of fused cells secreting antibody of predefined specificity. Nature. 1975;256:495-7.

21. Akobeng AK. Understanding diagnostic tests 3: receiver operating characteristic curves. Acta Paediatr. 2007;96:644-7.

22. Zhang YS, Chu JH, Cui SX, et al. Des- $\gamma$-carboxy prothrombin (DCP) as a potential autologous growth factor for the development of hepatocellular carcinoma. Cell Physiol Biochem. 2014;34:903-15.

23. Manuc D, Preda CM, Sandra I, et al. Signification of serum alpha-fetoprotein levels in cases of compensated cirrhosis and hepatitis C virus without hepatocellular carcinoma. J Med Life. 2020;13:68-74.

24. Arai S, Miyazaki T. A scavenging system against internal pathogens promoted by the circulating protein apoptosis inhibitor of macrophage (AIM). Semin Immunopathol. 2018;40:567-75.

25. Okanoue T, Itoh $\mathrm{Y}$, Minami M, et al. Interferon therapy lowers the rate of progression to hepatocellular carcinoma in chronic hepatitis $\mathrm{C}$ but not significantly in an advanced stage: a retrospective study in 1148 patients. J Hepatol. 1999;30:653-9.

26. Sugisawa R, Komatsu G, Hiramoto E, et al. Independent modes of disease repair by AIM protein distinguished in AIM-felinized mice. Sci Rep. 2018;8:13157.

27. Yamazaki T, Mori M, Arai S, et al. Circulating AIM as an indicator of liver damage and hepatocellular carcinoma in humans. PLoS ONE. 2014;9:e109123.

Publisher's Note Springer Nature remains neutral with regard to jurisdictional claims in published maps and institutional affiliations. 